\title{
First Mode Schumann Resonance Frequency Variation During a Solar Proton Event
}

\author{
Minu Sanfui and Debasish Biswas* \\ Department of Physics, Jadavpur University, Kolkata, India
}

Received 12 June 2014, revised 17 November 2015, accepted 2 December 2015

\begin{abstract}
A severe X-ray flare occurred on 06 - 07 March 2012 followed by a solar proton event (SPE). During this event we studied the variation in frequency of the first Schumann resonance (SR) spectra mode from the recorded data over Kolkata $\left(22.56^{\circ} \mathrm{N}, 88.5^{\circ} \mathrm{E}\right)$. The first mode frequency enhanced $(\sim 8.14 \mathrm{~Hz}, 3.85 \%)$ during the solar X-ray bursts and immediately after its value decreased $(\sim 7.44 \mathrm{~Hz}, 5.13 \%)$ during the proton event. The influences of SPE and X-ray bursts upon the SR frequency fluctuation are explained in terms of the changes in medium ionization, i.e., the change in dielectric property and two layer reflection height variation in the waveguide. The geomagnetic storm effect on the modification of this frequency variation occurring during that time is also considered.
\end{abstract}

Key words: Earth-ionosphere cavity, Solar flare, Schumann resonance, Medium conductivity, Two layer theory

Citation: Sanfui, M. and D. Biswas, 2016: First mode Schumann resonance frequency variation during a solar proton event. Terr. Atmos. Ocean. Sci., 27, 253-259, doi: 10.3319/TAO.2015.12.02.01(AA)

\section{INTRODUCTION}

The Schumann resonance (SR) is the electromagnetic resonance phenomenon generated by global lightning discharges within the resonator formed between the Earth and the ionosphere. The various aspects of SR phenomena are being explored (Balser and Wagner 1960; Madden and Thompson 1965; Ogawa et al. 1969; Sao et al. 1973; Tanahashi 1976; Sentman 1983; Williams 1992; Füllekrug 1995; Füllekrug and Fraser-Smith 1996; Yampolski et al. 1997; Heckman et al. 1998; Nickolaenko et al. 1998, 1999; Balling and Hildebrandt 2000; Hobara et al. 2000; Shvets 2001; Nickolaenko and Hayakawa 2002, 2007; Sekiguchi et al. 2006) over the years. The seasonal and day-to-day variability in SR intensities, frequencies, and quality factors are reported with possible explanations for such changes (Balser and Wagner 1962; Galejs 1970; Cannon and Rycroft 1982; Nickolaenko and Rabinowicz 1995; Sátori 1996; Roldugin et al. 1999, 2001, 2003, 2004; Price and Melnikov 2004; Melnikov et al. 2004; Sekiguchi et al. 2008). These changes are interpreted mainly by the influence of the temporal and spatial variations in the inhomogeneity and conductivity of the medium along with the variations in location and inten-

\footnotetext{
* Corresponding author

E-mail:debasish.juphys@gmail.com
}

sities of the three global thunderstorm generating centers. The changes in ionization in the lower ionosphere with that of zenith angle of the Sun along with the signal propagation path from lightning flashes to the point of observations are also responsible for this process. The inhomogeneities originate from the asymmetry of the day- and night-time ionosphere. Another class of inhomogeneity may arise due to unequal extra-ionization in different layers during solar $\mathrm{X}$-ray flares (SF) and solar proton events (SPE). SF cause extra-ionization mainly in the $70-80 \mathrm{~km}$ height range, whereas SPE can cause extra-ionization up to a depth of $40-50 \mathrm{~km}$. The effect of this kind of inhomogeneity on SR can be explained using the two-layer waveguide model (Greifinger and Greifinger 1978).

The specific linkage between the SR peak frequency values with global and regional temperatures is well known. The variation in SR peak frequency has been proven to be useful in indicating the variations in the tropical and global temperature (Williams 1992; Balling and Hildebrandt 2000).

The variation in SR frequency is related to the thunderstorm distribution and may be used as an indicator of thunderstorm source distribution. SR frequency variations have been established as an indicator of the thunderstorm 
distribution over the globe (Nickolaenko and Rabinowicz 1995). Diurnal and seasonal variations in SR parameters like amplitude, frequency, and Q-factor are explored (Price and Melnikov 2004; Sekiguchi et al. 2008). The parametric measurements of SR occurrences can be used to study the inter-annual variability in global thunderstorm activity centers in the three different continental regions (Price and Melnikov 2004). The maximum frequency variation of about $0.3,0.4$, and $0.7 \mathrm{~Hz}$ was observed in the first, second and third mode respectively and has been explained in terms of the ionospheric electron density variation in the Himalayan region, India (Chand et al. 2009).

Because of the geomagnetic field, the anisotropic conductivity of the cavity influences the wave propagation in the Earth-ionosphere cavity. Within the cavity disturbances like random fluctuations of irregularities are occurring regularly along with other causalities, such as Polar-Cap absorption, X-ray bursts, etc. The inhomogeneity and anisotropy of the cavity are supposed to introduce line splitting of the SR spectra (Bliokh et al. 1980; Sentman 1989; Labendz 1998). Vertical profiles of the electric and magnetic fields of the first global SR in the Earth-ionosphere cavity are investigated numerically with various vertical profiles of the ionospheric electron concentration (Grimalsky et al. 2005).

Solar proton precipitation introduces an abrupt frequency variation. It is enhanced by the interaction between the polar ionosphere and the precipitating particles. It causes the whole polar ionosphere to shift downward causing the first SR mode frequency to move globally to lower values (Schlegel and Füllekrug 1999; Roldugin et al. 1999, 2001, 2003).

Protons with energy up to $100 \mathrm{MeV}$ are most often emitted from the Sun in conjunction with solar flares, which can penetrate deeply into the D-region and cause additional ionization leading to the conductivity changes thereby modifying the SR parameters (Roldugin et al. 1999, 2001, 2003). High solar proton fluxes cause serious radiation hazards in the solar system (Reedy 2006). The spectra of the signals in the realistic and idealistic Earth-ionosphere cavities have been computed in the frequency and time domains and the features pertinent to the Earth-ionosphere cavity with exclusively low losses implying a special atmospheric conductivity model are discussed (Nickolaenko and Hayakawa 2014).

The variation in frequency of the first SR spectra mode during SPE from 06 - 07 March 2012 is presented in this paper from the observed data recorded over Kolkata. The result shows the decrease in frequency during SPE occurrence and enhancement of the first mode frequency during the severe solar X-ray bursts occurring just before the proton event. The influences of SPE and X-ray bursts upon the SR frequency fluctuation are explained in terms of changes in ionization produced by the events and corresponding modification in the Earth-ionosphere waveguide.

\section{EXPERIMENTAL SET-UP}

The antenna system for these experiments were erected on the ground at a vast bare land area near Kolkata having the geographic coordinates (Lat: $22.56^{\circ} \mathrm{N}$, Long: $88.5^{\circ} \mathrm{E}$ ).

Square-loop antennas were made for the detection of extremely low frequency (ELF) signals. Two such loops were mounted onto wooden structures connected in series and thus their effective gain was increased beyond $5 \mathrm{~dB}$. The length of each side of the square loops is $1 \mathrm{~m}$ with a total of 75 turns and the other with 90 turns has the length of $1.3 \mathrm{~m}$ for each side. A coaxial cable $50 \mathrm{~m}$ in length is used to transfer the signal from the antenna into the receiver input where it is pre-amplified. A stereo-preamplifier-integrated circuit with LA3161 chip is chosen whose low frequency response starts below $5 \mathrm{~Hz}$ and extends up to a few $\mathrm{kHz}$. This chip is superior to others in handling input voltage in the range of few microvolts to millivolts. The frequency selective stages were designed with active circuit elements. A wide-band amplifier whose output is taken through an active low-pass filter having cut-off frequency nearly $35 \mathrm{~Hz}$ further amplifies it. A wideband, very low input voltage sensitive and low frequency sensitive receiver was fabricated to detect input signals from $100-500 \mathrm{mV}$.

The detected ELF signal is sampled at a rate of 40000 times per second and the voltage is then converted into a 12-bit digital signal using a computerized data acquisition system through a PCI 1050, 16 channel 12 bit DAS card. These signals are further processed and stored in a computer. Because of the choice of sampling frequencies, no information is lost in the sampling process, except for the quantization error. The loop antennae are vertically mounted in series with their planes kept parallel to each other along a fixed $\mathrm{E}-\mathrm{W}$ or N-S direction. The recorded data were analyzed through Origin 5.0 software.

\section{RESULTS AND DISCUSSION}

High energy solar proton flux of 07 March 2012 was detected using the GOES 13 satellite at 0010 UT which showed solar proton maxima at 1500 UT. It was preceded by the solar X-ray bursts. Its onset occurred at about $0000 \mathrm{UT}$ and the maximum took place at $0010 \mathrm{UT}$. The variations in proton flux at energy $(E) \geq 100 \mathrm{MeV}$ and $\mathrm{X}$-ray at wavelength $(\lambda)$ at $0.1<\lambda<0.8 \mathrm{~nm}$ are shown in Fig. 1 by a continuous line curve and dashed line curve, respectively.

The changes in the first SR frequency on 07 March 2012 over Kolkata are shown by the continuous line graph joining the square blocks in Fig. 2. The values of the first SR frequency are averaged over five days adjacent to the day of occurrence for these two events. The frequencies are plotted by a dot-dashed curve. The SR frequency starts enhancing from 2300 UT March 6 and reaches the highest value $~ 8.14 \mathrm{~Hz}$ $(3.85 \%)$ at 0010 UT during solar bursts. Immediately after 
the solar proton flare, its value approaches a lower value showing the minima at $\sim 7.44 \mathrm{~Hz}(5.13 \%)$ because of the instantaneous high energy SPE. The decrease in the first mode SR frequency continued up to around 1500 UT and returned to its normal value at 2000 UT of March 7.

The earliest ELF propagation parameter models in the Earth-ionosphere waveguide were based on the supposition that the lower ionosphere conductivity profile is horizontally homogeneous and varies exponentially in the vertical direction. During solar activity there may be two kinds of changes: (1) increase in ionization level at the normal D-region reflection heights, and (2) the variation of D-region's lower edge (Roldugin et al. 1999). According to this model the variation in the first SR frequency is given by (Roldugin et al. 1999)

$$
\frac{\Delta f_{1}}{f_{1}} \approx 0.36\left(\frac{\Delta N}{2 N}+\frac{\Delta h}{h}\right)
$$

where $f_{1}$ is the first mode frequency, $\Delta f_{1}$ change in first mode frequency, $N$ is the ion density and $\Delta N$ change in ion density, $h$ is the ionospheric reflection height and $\Delta h$ change in reflection height.

This expression shows that the frequency can change due to both electron density variations in the ionospheric $\mathrm{D}$ region and the change in D-region lower boundary height. $\mathrm{X}$-ray flux growth enhances the electron density without a significant change in the ionospheric altitude, causing the first SR frequency increase. Solar protons penetrating deep into the atmosphere cause a decrease in D-region altitude.

\section{6 - 07 March 2012}

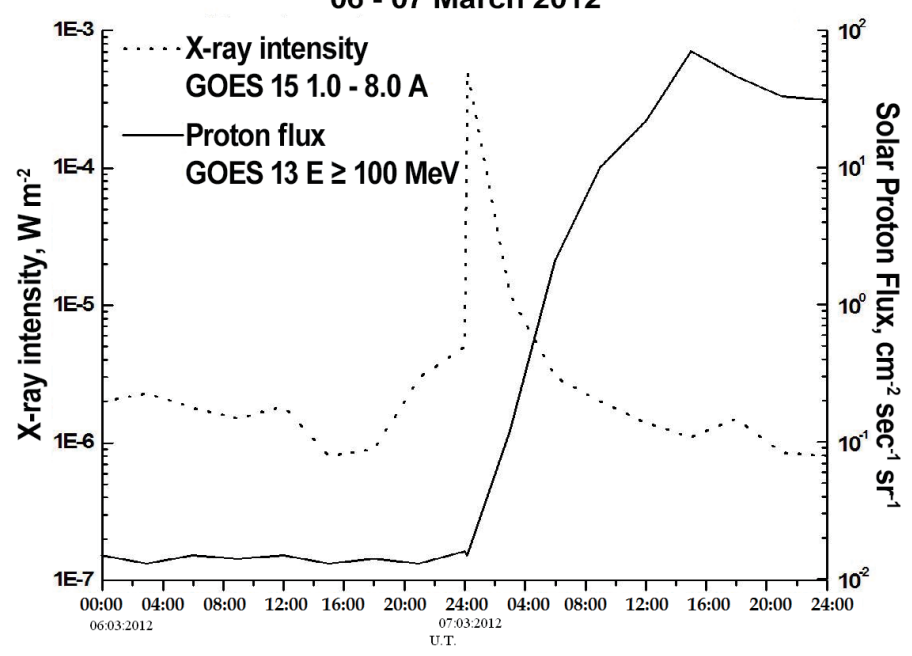

Fig. 1. Energetic solar proton flux and X-ray bursts as recorded onboard GOES 13 satellite during the solar proton event (SPE) of 06 - 07 March 2012. The continuous line curve is for the proton flux while the dashed line curve represents X-ray bursts.

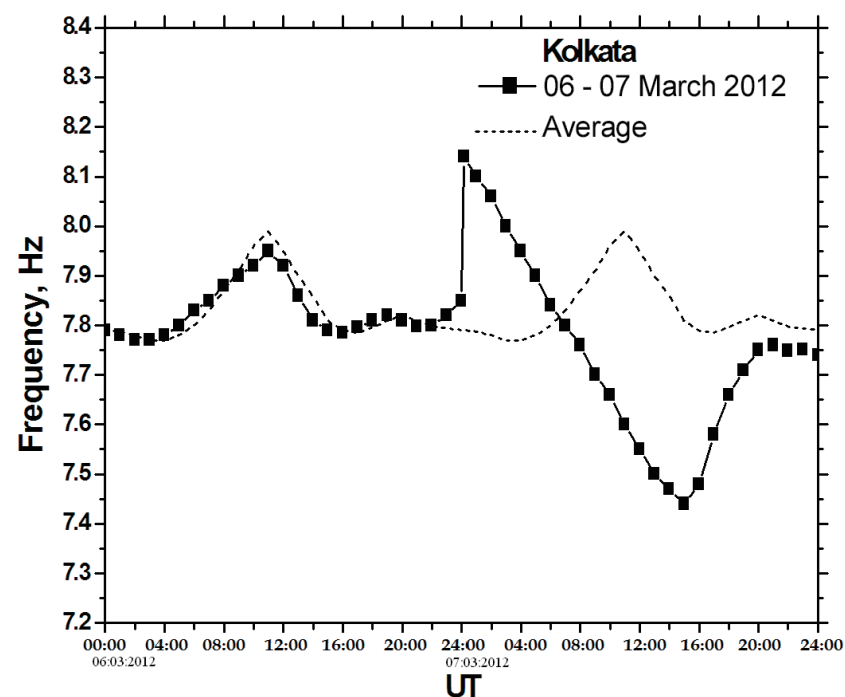

Fig. 2. Variation of the first mode SR frequency over Kolkata $\left(22.56^{\circ} \mathrm{N}, 88.5^{\circ} \mathrm{E}\right)$ on $06-07$ March 2012 depicted by the continuous line curve joining the square blocks. SR frequencies averaged over five days adjacent to the day of occurrence are shown. 
This gives rise to a decrease in the first SR frequency. SPE can also affect ionization. Thus, the two terms compete with each other and their relative magnitudes are the decisive factors, i.e., whether there will be an increase or decrease in the first SR frequency. If the two terms are comparable there may be no change in the first SR frequency. Apart from this, the two terms are inter-linked. This model will not be efficient in estimating the magnitude of variation in the first SR frequency. Later on, two characteristic layers were identified within the lower ionosphere (Madden and Thompson 1965) responsible for vertical electric field height variation and horizontal magnetic field components. Another kind of inhomogeneity can arise during solar Xray or proton flux enhancement (Greifinger and Greifinger 1978). In this method the eigenvalue depends on the conductivity of the atmosphere in the two height-independent characteristic layers: $h_{1}$ and $h_{2}$. One layer is defined as the height $h_{1}$ where displacement and conduction currents are equal (typically $40-55 \mathrm{~km}$ for $f<50 \mathrm{~Hz}$ ). The other is at a height of $60-70 \mathrm{~km}$, where $4 \mu_{0} \omega \sigma \zeta^{2}=1, \zeta$ being the conductivity scale height at the altitude responsible for ELF wave reflection. The unequal ionization of these two layers by solar extra-radiation can give rise to vertical inhomogeneity affecting the SR frequency. An approximate variation in medium conductivity around $30-80 \mathrm{~km}$ is shown in Fig. 3 (Sentman 1983). A modeled altitude profile of conductivity using an exponential equation under perturbation due to extra ionization of the medium can be expressed as (Sentman 1983)

$\sigma(z)=10^{-16} \exp \left[\frac{z}{3.1}+2.303 \beta \exp -\left(\frac{z-z_{0}}{a}\right)^{2}\right]$

where $\sigma(z)$ is the conductivity profile, the perturbation parameter $\beta$ is the perturbation amplitude in powers of ten about its undisturbed value, $z$ is the altitude and $z_{0}$ is a particular altitude, and $a$ is the width of perturbation.

According to the two layer SR theory frequencies can be expressed as (Sentman 1990)

$f_{n}=\sqrt{\frac{n(n+1)}{2 \pi}} \frac{c}{r_{e}} \sqrt{\frac{h_{1}}{h_{2}}}$

where $n$ is the mode number ( $n=1$, the first harmonic is regarded here), $c$ the velocity of light, $r_{e}$ the mean radius of the earth and $h_{1}$ and $h_{2}$ the two characteristic heights in the D-region.

For $n=1, f_{1}=\sqrt{\frac{1}{\pi}} \frac{c}{r_{e}} \sqrt{\frac{h_{1}}{h_{2}}}$

During an x-ray flare the lower height $h_{1}$ is almost un- affected because it causes extra-ionization mainly around $70 \mathrm{~km}$, i.e., the height of reflection $h_{2}$ is slightly reduced by the value $\delta_{2}$ so that the modified first SR frequency becomes $\left(f_{1}\right)_{S R}=\sqrt{1 / \pi}\left(c / r_{e}\right) \sqrt{h_{1} /\left(h_{2}-\delta_{2}\right)}$. The first SR frequency, therefore, increases. In our observation the proton event was preceded by a flare during which we observed an increase in the first SR frequency. During the solar flare the reflectivity of the upper height is also increased. This fact contributes additionally to the increase in the first SR frequency. During a SPE energetic protons can penetrate deeply into the ionosphere up to $50 \mathrm{~km}$ and can ionize regions lower than the normal D-region (Roldugin et al. 1999; Clilverd et al. 2006). In this case $h_{1}$ changes by the value $\delta_{1}$. If the effect on $h_{2}$ is neglected the modified first SR frequency will be $\left(f_{1}\right)_{S P E}=\sqrt{1 / \pi}\left(c / r_{e}\right) \sqrt{\left(h_{1}-\delta_{1}\right) / h_{2}}$.

As a result, the first SR frequency decreases. The altitude profile in perturbation of the first SR frequency, using the perturbation parameter $\beta=3$ and the width parameter $a=6$ is shown in Fig. 4 along with its unperturbed profile $(\beta=0)$. The dashed curve in this figure depicts the first resonance frequency altitude profile using the FDTD / prony result (Sentman 1983). The geomagnetic storm is also an important agent that produces ionospheric disturbances and hence may also be responsible for generating transient electromagnetic pulses which in turn may enhance the SR signal levels when superposed in this frequency band. The geomagnetic storm observed during that period is evident from Fig. 5. Thus the observed signal variation, i.e., the SR frequency restoration almost to the undisturbed value may be due to the geomagnetic storm.

\section{CONCLUSION}

The variation in frequency of the first SR spectra mode is not dependent on source-ionosphere geometry but depends on the waveguide characteristics. This kind of variation is to be explained by the two-characteristic-layer-model of two different heights. For the purpose of modeling the variation in the first SR frequency associated with SPE and solar Xray bursts preliminary electron density data at the two characteristic heights are to be known during respective solar events. Further, very low frequency (VLF) phase and amplitude observations could be used to determine the ionospheric electron density variation. Very low frequency signals are known to be seriously affected during SPEs propagating through the Earth-ionosphere cavity at about $50-60 \mathrm{~km}$ altitude (Clilverd et al. 2006). The D-region electron density and ionization change during high energy particle precipitation down to this altitude depend upon the particle energy at this altitude. The correlation between solar activity and SR parameters might be used for many studies like the study of the Sun and extraterrestrial sources. Thus, it can be concluded that as the D-region and nearby layers are perturbed due to solar flares mainly by SPE and X-ray bursts, and the 


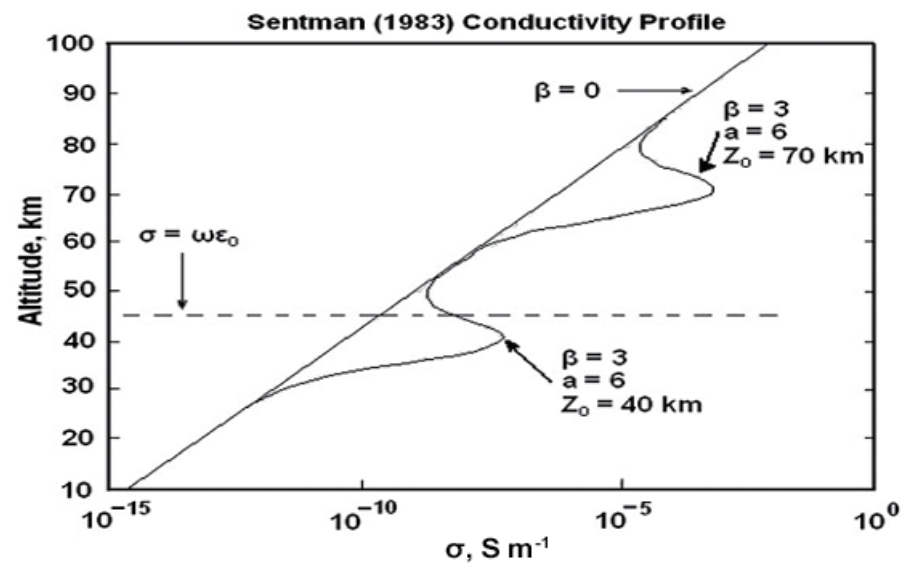

Fig. 3. Conductivity model according to Sentman (1983) using the exponential equation with perturbation parameter $\beta$. The line $\sigma=\omega \varepsilon_{0}$ signifies the altitude in which ionosphere becomes conductive.

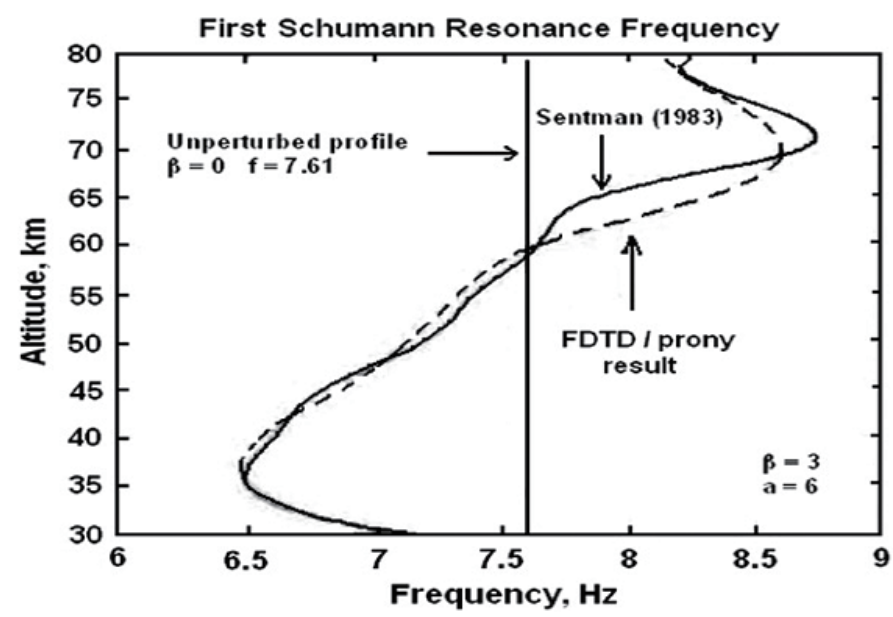

Fig. 4. Perturbed profile of first SR frequency according to Sentman (1983).

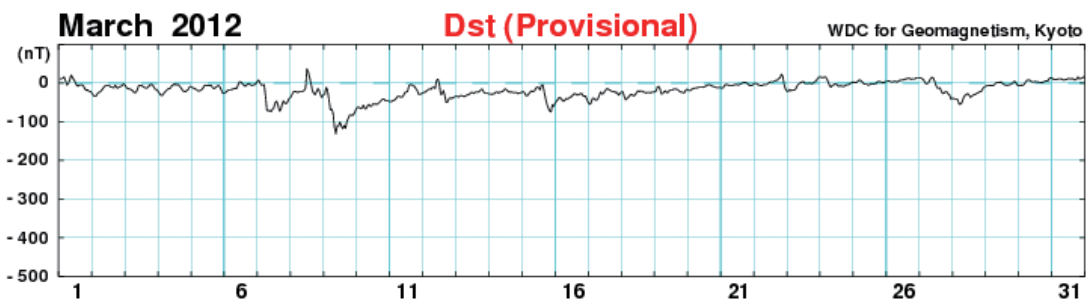

Fig. 5. Variations in Dst-index during the month of March, 2012 (http://wdc.kugi.kyoto-u.ac.jp/dst_provisional/). (Color online only)

corresponding modification of dielectric property and reflection height change the SR mode frequency. In this way we can detect these kinds of extra-terrestrial events comparing the continuous time series spectra of the SR frequencies with some specific previous signature.

Acknowledgements The authors are thankful to Prof. S. S. De (Retd.), Institute of Radio Physics and Electronics, University of Calcutta for his kind help and Dr. D. Haldar for stimulating discussions. The authors thank the reviewers for their assistance in improving the quality of the article.

\section{REFERENCES}

Balling, R. C. and M. Hildebrandt, 2000: Evaluation of the linkage between Schumann Resonance peak frequency values and global and regional temperatures. Clim. Res., 16, 31-36, doi: 10.3354/cr016031. [Link] 
Balser, M. and C. A. Wagner, 1960: Observations of earthionosphere cavity resonances. Nature, 188, 638-641, doi: 10.1038/188638a0. [Link]

Balser, M. and C. A. Wagner, 1962: On frequency variations of the Earth-ionosphere cavity modes. J. Geophys. Res., 67, 4081-4083, doi: 10.1029/JZ067i010p04081. [Link]

Bliokh, P. V., A. P. Nickolaenko, and I. F. Fillippov, 1980: Schumann resonances in earth -ionosphere cavity. IEE Electromagnetic Waves Series, Volume 9, Peter Peregrinus Ltd., Stevenage, Herts, England, p175.

Cannon, P. S. and M. J. Rycroft, 1982: Schumann resonance frequency variations during sudden ionospheric disturbances. J. Atmos. Terr. Phys., 44, 201-206, doi: 10.1016/0021-9169(82)90124-6. [Link]

Chand, R., M. Israil, and J. Rai, 2009: Schumann resonance frequency variations observed in magnetotelluric data recorded from Garhwal Himalayan region India. Ann. Geophys., 27, 3497-3507, doi: 10.5194/ angeo-27-3497-2009. [Link]

Clilverd, M. A., A. Seppälä, C. J. Rodger, N. R. Thomson, P. T. Verronen, E. Turunen, T. Ulich, J. Lichtenberger, and P. Steinbach, 2006: Modeling polar ionospheric effects during the October-November 2003 solar proton events. Radio Sci., 41, RS2001, doi: 10.1029/2005RS003290. [Link]

Füllekrug, M., 1995: Schumann resonances in magnetic field components. J. Atmos. Terr. Phys., 57, 479-484, doi: 10.1016/0021-9169(94)00075-Y. [Link]

Füllekrug, M. and A. C. Fraser-Smith, 1996: Further evidence for a global correlation of the Earth-ionosphere cavity resonances. Geophys. Res. Lett., 23, 2773-2776, doi: 10.1029/96GL02612. [Link]

Galejs, J., 1970: Frequency variations of Schumann resonances. J. Geophys. Res., 75, 3237-3251, doi: 10.1029/ JA075i016p03237. [Link]

Greifinger, C. and P. Greifinger, 1978: Approximate method for determining ELF eigenvalues in the earthionosphere waveguide. Radio Sci., 13, 831-837, doi: 10.1029/RS013i005p00831. [Link]

Grimalsky, V., S. Koshevaya, A. Kotsarenko, and R. P. Enriquez, 2005: Penetration of the electric and magnetic field components of Schumann resonances into the ionosphere. Ann. Geophys., 23, 2559-2564, doi: 10.5194/angeo-23-2559-2005. [Link]

Heckman, S. J., E. Williams, and B. Boldi, 1998: Total global lightning inferred from Schumann resonance measurements. J. Geophys. Res., 103, 31775-31779, doi: 10.1029/98JD02648. [Link]

Hobara, Y., N. Iwasaki, T. Hayashida, N. Tsuchiya, E. R. Williams, M. Sera, Y. Ikegami, and M. Hayakawa, 2000: New ELF observation site in Moshiri, Hokkaido Japan and the results of preliminary data analysis. $J$. Atmos. Electr., 20, 99-109.
Labendz, D., 1998: Investigation of Schumann resonance polarization parameters. J. Atmos. Sol.-Terr. Phys., 60, 1779-1789, doi: 10.1016/S1364-6826(98)00152-7. [Link]

Madden, T. and W. Thompson, 1965: Low-frequency electromagnetic oscillations of the Earth-ionosphere cavity. Rev. Geophys., 3, 211-254, doi: 10.1029/ RG003i002p00211. [Link]

Melnikov, A., C. Price, G. Sátori, and M. Füllekrug, 2004: Influence of solar terminator passages on Schumann resonance parameters. J. Atmos. Sol.-Terr. Phys., 66, 1187-1194, doi: 10.1016/j.jastp.2004.05.014. [Link]

Nickolaenko, A. P. and M. Hayakawa, 2002: Resonances in the Earth-Ionosphere Cavity, Modern Approaches in Geophysics, Vol. 19, Springer Science \& Business Media, New York, 380 pp.

Nickolaenko, A. P. and M. Hayakawa, 2007: Recent studies of Schumann resonance and ELF transients. J. Atmos. Electr., 27, 19-39.

Nickolaenko, A. P. and M. Hayakawa, 2014: Spectra and waveforms of ELF transients in the Earth-ionosphere cavity with small losses. Radio Sci., 49, 118-130, doi: 10.1002/2013RS005281. [Link]

Nickolaenko, A. P. and L. M. Rabinowicz, 1995: Study of the annual changes of global lightning distribution and frequency variations of the first Schumann resonance mode. J. Atmos. Terr. Phys., 57, 1345-1348, doi: 10.1016/0021-9169(94)00114-4. [Link]

Nickolaenko, A. P., G. Sátori, B. Zieger, L. M. Rabinowicz, and I. G. Kudintseva, 1998: Parameters of global thunderstorm activity deduced from the long-term Schumann resonance records. J. Atmos. Sol.-Terr. Phys., 60, 387-399, doi: 10.1016/S1364-6826(97)00121-1. [Link]

Nickolaenko, A. P., M. Hayakawa, and Y. Hobara, 1999: Long-term periodical variations in global lightning activity deduced from the Schumann resonance monitoring. J. Geophys. Res., 104, 27585-27591, doi: 10.1029/1999JD900791. [Link]

Ogawa, T., Y. Tanaka, and M. Yasuhara, 1969: Schumann resonances and worldwide thunderstorm activity--Diurnal variations of the resonant power of natural noises in the earth-ionosphere cavity. J. Geomagn. Geoelectr., 21, 447-452, doi: 10.5636/jgg.21.447. [Link]

Price, C. and A. Melnikov, 2004: Diurnal, seasonal and inter-annual variations in the Schumann resonance parameters. J. Atmos. Sol.-Terr. Phys., 66, 1179-1185, doi: 10.1016/j.jastp.2004.05.004. [Link]

Reedy, R. C., 2006: Solar-proton event-integrated fluences during the current solar cycle. $37^{\text {th }}$ Annual Lunar and Planetary Science Conference, March 13-17, 2006, League City, Texas, abstract no.1419.

Roldugin, V. C., Y. P. Maltsev, A. N. Vasiljev, and E. V. Vashenyuk, 1999: Changes of the first Schumann 
resonance frequency during relativistic solar proton precipitation in the 6 November 1997 event. Ann. Geophys., 17, 1293-1297, doi: 10.1007/s00585-999-12939. [Link]

Roldugin, V. C., Y. P. Maltsev, G. A. Petrova, and A. N. Vasiljev, 2001: Decrease of the first Schumann resonance frequency during solar proton events. J. Geophys. Res., 106, 18555-18562, doi: 10.1029/2000JA900118. [Link]

Roldugin, V. C., Y. P. Maltsev, A. N. Vasiljev, A. V. Shvets, and A. P. Nikolaenko, 2003: Changes of Schumann resonance parameters during the solar proton event of 14 July 2000. J. Geophys. Res., 108, 1103, doi: 10.1029/2002JA009495. [Link]

Roldugin, V.C., Y.P. Maltsev, A.N. Vasiljev, A. Y. Schokotov, and G. G. Belyajev, 2004: Schumann resonance frequency increase during solar X-ray bursts. J. Geophys. Res., 109, A01216, doi: 10.1029/2003JA010019. [Link]

Sao, K., M. Yamashita, S. Tanahashi, H. Jindoh, and K. Ohta, 1973: Experimental investigations of Schumann resonance frequencies. J. Atmos. Terr. Phys., 35, 20472053, doi: 10.1016/0021-9169(73)90118-9. [Link]

Sátori, G., 1996: Monitoring Schumann resonances--II. Daily and seasonal frequency variations. J. Atmos. Terr. Phys., 58, 1483-1488, doi: 10.1016/0021-9169(95)00146-8. [Link]

Schlegel, K. and M. Füllekrug, 1999: Schumann resonance parameter changes during high-energy particle precipitation. J. Geophys. Res., 104, 10111-10118, doi: 10.1029/1999JA900056. [Link]

Sekiguchi, M., M. Hayakawa, A. P. Nickolaenko, and Y. Hobara, 2006: Evidence on a link between the inten- sity of Schumann resonance and global surface temperature. Ann. Geophys., 24, 1809-1817, doi: 10.5194/ angeo-24-1809-2006. [Link]

Sekiguchi, M., Y. Hobara, and M. Hayakawa, 2008: Diurnal and seasonal variations in the Schumann resonance parameters at Moshiri, Japan. J. Atmos. Electr., 28, 1-10.

Sentman, D. D., 1983: Schumann resonance effects of electrical conductivity perturbations in an exponential atmospheric/ionospheric profile. J. Atmos. Terr. Phys., 45, 55-65, doi: 10.1016/S0021-9169(83)80008-7. [Link]

Sentman, D. D., 1989: Detection of elliptical polarization and mode splitting in discrete Schumann resonance excitations. J. Atmos. Terr. Phys., 51, 507-519, doi: 10.1016/0021-9169(89)90090-1. [Link]

Sentman, D. D., 1990: Approximate Schumann resonance parameters for a two-scale-height ionosphere. $J$. Atmos. Terr. Phys., 52, 35-46, doi: 10.1016/0021-9169(90)90113-2. [Link]

Shvets, A. V., 2001: A technique for reconstruction of global lightning distance profile from background Schumann resonance signal. J. Atmos. Sol.-Terr. Phys., 63, 10611074, doi: 10.1016/S1364-6826(01)00024-4. [Link]

Tanahashi, S., 1976: Detection of line splitting of Schumann resonances from ordinary data. J. Atmos. Terr. Phys., $\mathbf{3 8}$, 135-142, doi: 10.1016/0021-9169(76)90120-3. [Link]

Williams, E. R., 1992: The Schumann resonance: A global tropical thermometer. Science, 256, 1184-1187, doi: 10.1126/science. 256.5060.1184. [Link]

Yampolski, Y. M., P. V. Bliokh, V. S. Beley, V. G. Galushko, and S. B. Kascheev, 1997: Non-linear interaction between Schumann resonances and HF signals. J. Atmos. Sol.-Terr. Phys., 59, 335-342, doi: 10.1016/ S1364-6826(96)00036-3. [Link] 